\title{
Lógicas y dialécticas trascendentales de la inversión en sectores de infraestructura empresarial en Colombia
}

\begin{abstract}
Abel María Cano Morales*
Resumen

El presente trabajo tiene dos objetivos primordiales: el primero busca identificar y discutir los temas que afectan el nivel y el patrón de las inversiones de las empresas de servicios públicos regulados especialmente en un departamento de Colombia, para ello se toma en cuenta la referencia nacional; el segundo objetivo trata de ilustrar de una manera simple y completa los efectos macroeconómicos y distributivos que estas inversiones tienen sobre otros sectores de la economía y sobre los núcleos familiares.

Para abordar el primer objetivo se hace necesario elaborar un modelo de inversiones en una empresa de servicios regulada, poniendo énfasis en la interacción entre modelo regulatorio e incentivos a la eficiencia, la eficacia y el capital deseado a largo plazo. Surge asi una interacción entre modelo regulatorio (método PRICE-CAP ${ }^{l}$ o ROR ${ }^{2}$ y costo de capital, extensión de la concesión) y costo de capital de la empresa regulada. En relación con el segundo objetivo se hace referencia a los resultados de un estudio de equilibrio general computarizado, el cual muestra cómo las decisiones de mejorar la eficacia, la productividad y la eficiencia en los sectores regulados se difunden en la economía, y cómo modifican el patrón de organización industrial y el nivel de bienestar de los sectores de ingreso (se utiliza una matriz de contabilidad social de 1995/96).
\end{abstract}

Palabras clave: empresa regulada, efectos macroeconómicos, modelo regulatorio, eficiencia, eficacia, capital deseado y costo de capital.

* Profesor investigador de la Universidad de Medellín y de la Universidad de Antioquia. Correo electrónico: amcano@udem.edu.co

1 Precio supuesto o simulado para efectos de verificar la consistencia de la inversión ante una variación supuesta en el precio.

2 Rentabilidad Obtenida Real, bajo el supuesto del costo de capital, la cual permite determinar cómo la rentabilidad es directamente proporcional a la variación en precios supuestos.

No. 220, septiembre-diciembre 2006 
Abel María Cano Morales

\section{Introducción}

Como se mencionó, el propósito de este trabajo es doble: por un lado, se busca identificar y discutir diferentes temas que afectan el nivel y patrón de inversiones de las empresas de servicios públicos regulados; por otro, se intenta ilustrar algunos de los efectos que estas inversiones tienen sobre otros sectores de la economía, particularmente en lo que se refiere a efectos macro (cuantificados en un modelo de equilibrio general) y a efectos distributivos (mediante las obligaciones de servicio universal que muchas veces enfrentan los diversos proveedores de servicios públicos).

Los temas que condicionan el nivel y patrón de las inversiones en infraestructura quedan en gran medida definidos en el marco regulatorio que se especifica para estos sectores y por la forma y mecanismos de privatización que se implementan, según las políticas de estado.

Los mecanismos de privatización tienen un efecto importante sobre el comportamiento de las empresas en relación con inversiones por distintas vías, tales como estructura de capital y reglas de finalización de las concesiones.

Un primer punto que caracteriza las privatizaciones en Colombia es la venta de paquetes de control de las empresas con requerimientos de operadores técnicos (caso concreto el que estamos viviendo actualmente con la venta parcial de Telecom), ya sea como parte de la sociedad inversora o con un contrato de servicios. Esta modalidad difiere de la venta de acciones en los mercados de capitales que caracterizó, por ejemplo, las privatizaciones en Chile y Gran Bretaña. Si bien esta forma de privatización reduciría el problema del principal agente asociado a la flotación de las acciones en la bolsa de valores ${ }^{3}$, tiene costos en términos de reducción de la competencia en el mercado de capitales (no hay riesgos de toma de posesión) y puede afectar las inversiones mediante un cambio forzado en la composición del capital (externo y local) como resultado de la necesidad de contar con algunos socios externos.

3 Sobre la importancia de este efecto en el caso chileno ver Bitrán, Hacia el estado regulador CIEPLAN [1995]. La gestión privada y la inversión en el sector eléctrico chileno (LC/L.1070), septiembre de 1997. 
Lógicas y dialécticas trascendentales de la inversión en sectores de infraestructura empresarial en Colombia

Un segundo punto que tiene impactos —que si bien no son inmediatos- de indudable importancia sobre el comportamiento de la inversión en estos sectores de infraestructura es la regla de finalización de las concesiones. A medida que se aproxima la finalización de una concesión aparecen incentivos para el titular del servicio para no invertir e incluso disminuir el mantenimiento de las redes existentes, en tanto no esté seguro de poder recuperar esa inversión en lo que resta de la concesión. En este punto analizaremos las reglas de terminación y compensación existentes en los distintos sectores con el fin de identificar posibles problemas en las mismas.

\section{Lógicas y dialécticas trascendentales de la inversión en sectores de infraestructura empresarial en Colombia}

En la reestructuración de los servicios públicos de infraestructura en Colombia se observa un alto grado de homogeneidad en cuanto a la búsqueda de mecanismos de mercado y la participación del sector privado como posible inversionista; por su parte, la regulación presenta marcadas variaciones entre sectores, existiendo un amplio rango de leyes y regímenes regulatorios.

Estas normas regulatorias afectan la inversión por medio de distintos mecanismos como el costo de capital, las obligaciones de servicios de las empresas (con respecto a actividades por las que es responsable, calidad, expansión, diversificación, entre otras), el tratamiento de relaciones intersectoriales (particularmente en relación con sectores que presentan una alta sustituibilidad o complementariedad), entre otros.

Un primer análisis, entonces, es el impacto que distintos regímenes regulatorios tienen sobre el riesgo enfrentado por la empresa, condicionando el costo de capital de las mismas. En un trabajo publicado por el Banco Mundial, Ian Alexander, Colin Mayer y Helen Weeds ${ }^{4}$ cuantifican distintos grados de riesgo asociado a cada régimen regulatorio. Estos autores encuentran que la regulación por precios máximos, si bien provee mayores incentivos a la eficiencia productiva, viene acompañada de un mayor riesgo de mercado (traducido en mayores betas) que la regulación por costo del servicio.

\footnotetext{
4 Véase Ian Alexander, Colin Mayer y Helen Weeds: Regulatory Structure Risk and Infraestructure Firms, Policy Research Working Paper 1998 - The World Bank - Private Sector Development Department December 1996. MAYER y WEEDS, Banco Mundial: Las reformas estructurales y la inversión privada en áreas de infraestructura (LC/L.1083), Chile, noviembre de 1997.
} 
Utilizando la información presentada por estos autores, conjuntamente con datos locales de intensidad de capital y estructura de financiamiento elaborados en forma propia, se estima el impacto que los regímenes regulatorios adoptados en los distintos sectores de infraestructura tienen sobre el costo de capital (como resultado del mayor riesgo) y la inversión de estos sectores.

En algunos sectores de infraestructura, la determinación de tarifas de los servicios que fueron fijadas durante un proceso de privatización cubre sólo la operación y mantenimiento de los activos existentes en algunos casos específicos (como el sucedido en Transporte de Energía Eléctrica y Servicio de Telefonía Nacional e Internacional) estableciéndose mecanismos ad hoc para la decisión y el financiamiento de las nuevas inversiones.

De esta forma, se ha introducido un cierto grado de separación entre la responsabilidad por la operación y mantenimiento y las decisiones de inversiones en el sistema. Esto lleva a que aparezca una potencial distorsión en la asignación de recursos debido a que las decisiones se realizan en forma separada y, por lo tanto, no se minimiza el costo total de provisión del sistema.

Al mismo tiempo, aparecen incentivos cruzados para que la otra parte afronte el costo. Las obligaciones de servicio y la universalidad, presentes en la mayoría de los contratos de concesión y/o licencias de servicios públicos, tienen también un impacto importante en la política de inversión de las mismas. Por un lado, las obligaciones de servicio expresadas en servicio obligatorio comprometen a las empresas a invertir en áreas potencialmente no rentables; por otro, si estas obligaciones están asociadas a determinados plazos de cumplimiento influyen el ritmo de inversiones, lo que en presencia de costos de ajuste puede tener un impacto no superficial sobre el costo de la inversión.

La importancia de las obligaciones de servicio en los servicios públicos abarca más allá del impacto que éstas tienen sobre el comportamiento de la empresa proveedora. El acceso a los servicios en condiciones y precios razonables constituyen un elemento clave de la calidad de vida de toda la población tanto por los efectos directos como por las fuertes externalidades que existen en muchos de estos sectores. 
Lógicas y dialécticas trascendentales de la inversión en sectores de infraestructura empresarial en Colombia

Las obligaciones de servicio universal (OSU) planteadas - tanto en lo relacionado con planes de cobertura como con los mecanismos de subsidios adoptados en la mayoría de los contratos de concesión - tienen un fuerte impacto sobre los sectores más pobres y necesitados de la población; por ello, en esta sección se analizarán algunas de las obligaciones de servicio existentes y su impacto sobre distintos estamentos de la población.

En primer lugar, se discute el efecto diferenciado de inversiones sobre las redes existentes, que surgen muchas veces de los requisitos de calidad mínima impuestos, y de inversiones en extensión de la red; mientras que las primeras benefician principalmente a usuarios existentes que en general pueden ser vistos como relativamente más ricos, las segundas tienen un fuerte impacto sobre el bienestar de los más pobres en tanto que son los que carecen totalmente de buenos servicios.

En segundo lugar, se analiza el impacto que tiene sobre la tarifa efectivamente pagada por los sectores más pobres los cargos por mora, desconexión y reconexión del servicio —eso sin contar los perjuicios a los que se ven sometidos cuando ocurre la desconexión— y la demora en que incurre la empresa para la reconexión del servicio. Esta diferencia entre tarifa nominal y la tarifa "efectiva" que pagan los usuarios más pobres como resultado de la incapacidad de afrontar a tiempo las facturas de los servicios públicos tiene importantes implicaciones sobre el nivel de bienestar de estos usuarios.

En tercer lugar, se discute el efecto que tiene sobre la parte de la población más pobre la introducción simultánea de varios servicios públicos. La falta de coordinación al evaluar voluntad y capacidad de pago por parte de los usuarios puede resultar en altas tasas de incobrabilidad de los servicios públicos ante la conexión simultánea de varios de ellos en barrios o estratos de bajos recursos.

Las profundas transformaciones que han sufrido gran parte de los servicios públicos y en los que se abrieron a la competencia y la participación privada sectores tradicionalmente monopólicos y de propiedad estatal tienen un impacto que abarca más allá del sector. Dada la importancia de estos sectores, la regulación adquiere un rol fundamental en términos de asegurar que las ganancias de eficiencia y de eficacia logradas por las empresas de servicios privatizadas sean trasladadas, al menos en una parte, a los usuarios finales y en especial a aquellos sectores menos favorecidos. 
En esta sección se analizará el impacto que tiene sobre la economía en su conjunto la eficiencia de la regulación de los distintos sectores de infraestructura. Utilizando un modelo de equilibrio general computado (MEGC) se cuantificarán los efectos de la regulación de los servicios públicos sobre el nivel de actividad y la tasa de ganancia relativa al nivel de 20 sectores productivos.

Dado que muchos de los sectores de infraestructura presentan fuertes interrelaciones tecnológicas (por ejemplo, entre agua y electricidad, electricidad y gas) y de sustitución en mercados finales (como electricidad y gas) la regulación de estos sectores debe ser consistente para evitar apropiación de rentas y distorsiones en el patrón de inversiones entre sectores. Con el fin de resaltar estas fuertes interrelaciones entre sectores, se realizará también un ejercicio para analizar el impacto de la regulación relativa y captura de renta y eficiencia entre sectores regulados.

Con el propósito de dar un marco analítico adecuado a estos problemas y poder explicitar las interrelaciones entre los distintos instrumentos regulatorios, en la sección II se presentará un modelo formal de inversiones en empresas reguladas.

Nuestro objetivo principal es apreciar cómo el régimen regulatorio y las cláusulas de un contrato de concesión determinan la política de inversión. Si pensamos en el contexto de un proceso de reestructuración y privatización, el problema del formulador de políticas es definir este conjunto de cláusulas del contrato de concesión, por ejemplo, duración del contrato, el régimen tarifario, las metas de inversión, la forma de financiamiento de la misma y los estándares mínimos de calidad de manera tal que permita maximizar el bienestar social.

La selección de instrumentos debe responder a los objetivos de sostenibilidad, eficiencia productiva, eficiencia asignativa y equidad de manera para que permita maximizar el bienestar social tanto en el corto como en el largo plazo. Un punto central de esta problemática es la existencia de "compensaciones" entre objetivos de manera tal que la mejora en uno de ellos muchas veces sólo puede ser lograda en detrimento de otro $u$ otros. 
Lógicas y dialécticas trascendentales de la inversión en sectores de infraestructura empresarial en Colombia

\section{Estructuración, diseño y presentación de un modelo de inversión aplicado en una empresa de servicios regulada}

Jorgenson y Handel sostenían, en un trabajo presentado en 1971, que

[...]para la industria regulada la teoría de acumulación óptima del capital es la misma que para las industrias reguladas, excepto que se impone una restricción adicional en la maximización del valor presente neto. Dado el precio del producto establecido por la agencia regulatoria, la cantidad de producto es determinada por el nivel de consumo público.

Esta visión del problema puede ampliarse significativamente a la luz de nuestros conocimientos actuales sobre las eventuales ganancias de eficiencia, eficacia y equidad, aunque igual puede suceder en términos de las compensaciones o tradeoffs implícitos entre la selección de regímenes tarifarios, el costo de capital y las obligaciones de servicio en cantidad, calidad y oportunidad.

Para comprender mejor esta apreciación, se presenta el proceso de maximización del valor presente neto de una firma que elige un plan de inversiones entre el presente y un momento del futuro $\mathrm{T}$, y que está sujeta al control de una agencia regulatoria, la cual se encarga de definir qué metas, restricciones e incentivos puede obtener una empresa, respetando la sostenibilidad y permanencia de la misma.

En términos generales supondremos que la empresa es neutral al riesgo aunque, como luego se podrá observar, para ello será necesario relajar un poco este resultado para poder discutir aspectos tales como el de costo de capital y de sostenibilidad.

El objetivo principal que se pretende desarrollar mediante este trabajo es el de apreciar cómo el régimen regulatorio y las cláusulas del contrato de concesión determinan la política de inversión. Si pensamos en el contexto de un proceso de reestructuración y privatización como el que se viene desarrollando en Colombia en estos últimos años, el problema del formulador de políticas es definir este conjunto de cláusulas del contrato de concesión de manera tal que permita maximizar el bienestar social de la población de 
menores ingresos. ${ }^{5}$ La duración del contrato, el régimen tarifarío, las metas de inversión, la forma de financiamiento de la misma y los estándares mínimos de calidad son, entre otros, los instrumentos disponibles al momento de definir la transformación de un sector.

Veamos algunos ejemplos que ilustran estos dilemas normativos.

- Un aumento de la proporción de la inversión directamente pagada por los usuarios $(\mho \vee \propto$ disminución de la inversión a ser recuperada por la vía tarifaría) (Po) resulta en una mayor inversión de equilibrio $\left(K^{*}\right)$ sin afectar la sostenibilidad de la empresa. Sin embargo, el efecto de esta política sobre el bienestar dependerá de la elasticidad ingreso (afectado por el aumento en el cargo de infraestructura - $\mathrm{m}$ ) y de la elasticidad precio (afectado directamente - Po) que tengan los consumidores.

- Los incentivos por invertir pueden ser mejorados extendiendo el plazo de la concesión $(T)$, lo que lleva a una compensación intergeneracional, pues al extender el plazo parte del costo de las inversiones actuales se trasladan a las generaciones futuras. Un efecto similar se asocia a un aumento en la compensación al terminar la concesión.

- La extensión del periodo del contrato $(T)$ mejora los incentivos para invertir, pero al mismo tiempo aumenta el costo de capital al incrementar el riesgo asociado a un contrato necesariamente más incompleto.

- Un régimen tarifario de alto poder de incentivos, mejora los incentivos a la eficiencia productiva, pero disminuye la cobertura $\left(K^{*}\right)$ y aumenta el costo de capital $(r)$.

\footnotetext{
5 Sobre la importancia de este efecto en el caso chileno véase Bitrán, op. cit. La determinación de una reestructuración óptima (en términos de separación vertical y horizontal de la empresa) sólo puede ser analizada en un contexto intersectorial que excede el comportamiento de la empresa objeto de análisis y que se intenta modelar aquí. Por lo tanto aspectos como la definición del alcance de cada actividad, la existencia o no de restricciones a la propiedad y/o participación en otras actividades, los efectos de reglas de regulación diferente entre sectores (como gas, electricidad, entre otros) deben necesariamente tratarse en un marco de equilibrio general que no son objeto del alcance de este trabajo.
} 
Lógicas y dialécticas trascendentales de la inversión en sectores de infraestructura empresarial en Colombia

La existencia de estas compensaciones entre objetivos es central al problema regulatorio, tanto en lo que hace al diseño del contrato original como a la forma en que éste es administrado durante su duración por la agencia reguladora. Una clara compresión de estos elementos y la forma en que interactúan resulta entonces esencial.

\section{Aproximaciones al modelo cotidiano para toma de decisiones de inversión en una empresa de servicios regulado (caso empresa colombiana)}

Para iniciar el proceso es necesario tomar como referencia un modelo tradicional de decisiones de inversión con costos de ajuste, sin regulación y aun con el precio (o tarifa) fijado exógenamente (por la presión de la competencia) la empresa determina su política de inversión para maximizar su valor presente neto $(V P N)$ :

Sujeto a:

$$
V P N=\int_{0}^{T}\left[p(\alpha) q-c(q, \alpha)-\phi I-\xi I^{2}\right] e^{r t d} t,
$$

1) $K^{\cdot}=I-\delta K$

2) $q \leq \gamma K$

En estas expresiones $q$ es la cantidad producida y vendida, independientemente del precio, pero que requiere de una disponibilidad mínima de capacidad por unidad de producto dada por la ecuación (2); en tanto que $K$ evoluciona según (1), donde $I$ es el monto de inversiones físicas brutas (del índice de bienes de capital) y $\delta$ es la tasa de amortización.

En la expresión de $V P N, T$ es el tiempo que la empresa planea estar en el mercado (determinado por ella misma), $p(\alpha)$ es el precio del producto unitario que se supone dependiente de un parámetro de calidad $\alpha$, de modo que:

$$
P \quad(\alpha) \geq 0
$$

La calidad esperada aquí se alcanza con mayores costos operativos, aunque podría plantearse una eventual sustitución entre $K$ y los costos operativos.

El costo de capital esta dado por $r$, que por ahora puede considerarse constante, pero que en general se compone de varios elementos: 


$$
\text { (3) } r=r_{f}+\beta\left(E_{M}-r_{f}\right)+\text { primadeval }+ \text { primadefaul }
$$

Es decir que el costo de capital es la suma equivalente a la tasa libre de riesgo ( $r f$ ) más la proporción de la prima de riesgo de mercado ( $E m$ - $r f$ ), que determina el grado de coparticipación que tiene esta industria con el mercado dado, más las primas de devaluación y por defecto si se admite la libre movilidad de capitales.

Los costos de inversión son de dos tipos: el precio corriente de los bienes de inversión $\varnothing$ por el índice de inversión $I$ da el gasto estimado en bienes de inversión si su oferta es completamente elástica; sin embargo, se admite que si los planes de inversión (nueva y de reposición) son demasiado ambiciosos (hacer las obras más rápido) pueden hacer que suban sustancialmente los costos en el término $\xi I^{2}$, donde $\xi$ es un parámetro positivo.

Admitiendo que la empresa no enfrenta indivisibilidades de $K$, que la obliguen a arrastrar con la capacidad ociosa, la condición (2) se cumplirá como una igualdad.

$2^{\prime} q=\gamma K$

Es decir $\gamma$ es la productividad media física del capital.

Esta hipótesis no es tan ingenua como parece. En muchos sectores regulados hay indivisibilidades marcadas del stock de capital, lo cual hace que se paguen altos costos de capital sobre activos inmovilizados por mala predicción de la demanda o porque la regulación obliga a satisfacerla de manera inmediata sin esperar que se superen umbrales de viabilidad económica.

En estas condiciones según el modelo hamiltoniano, el incremento del valor total de los activos de la empresa está dado por: ${ }^{6}$

$$
\text { (4) }\left[p(\alpha) \gamma K-C(\gamma K, \alpha)-\phi I-\xi I^{2}\right] e^{-r t}+\lambda(I-\delta K)
$$

\footnotetext{
6 Para el desarrollo de este modelo trabajamos con una hipótesis de subaditividad de costos de la firma, lo que es compatible con costos marginales crecientes y un único productor en el mercado. Alternativamente, se puede plantear un modelo con una hipótesis de monopolio natural tradicional, con costos marginales decrecientes o constantes, donde la escala viene determinada por la demanda. Aun con la hipótesis adoptada los costos fijos (fI) son un elemento crucial aunque la solución sea planteada en términos de costos marginales crecientes.
} 
Lógicas y dialécticas trascendentales de la inversión en sectores de infraestructura empresarial en Colombia

Donde $\lambda$ es el precio sombra del capital.

Las condiciones de máximo requieren entonces:

$$
\begin{aligned}
& \text { (5) } H_{d}=\left[p^{\prime}(\alpha) \gamma K-C_{\alpha}^{\prime}\right] e^{-r t}=0, \\
& \text { (6) } H_{i}=(\phi+2 \xi I) e^{-r t}+\lambda=0, \\
& \text { (7) } H_{k}+\lambda=e^{-r}\left[p(\alpha) \gamma-c_{q}^{\prime} \gamma\right]-\lambda \delta+\lambda=0,
\end{aligned}
$$

Más las condiciones de transversalidad:

$$
\text { (8) } H \quad(T \quad)=0
$$

Que indica que al momento de salirse de la industria, la empresa no ve incrementos futuros de valor de sus activos financieros y físicos y a su vez:

(9) $\lambda(T) K(T)=0$

$\lambda \quad(T) \geq 0$

Que indica que no se abandona capital inutilizado si su precio sombra es positivo.

Cuando la calidad no importa para el precio del producto que ve la empresa, es decir $p^{\prime}(\alpha)=0$, debe esperarse que $\alpha$ sea cero en la ecuación (5)

Por otra parte, las ecuaciones (6) y (7) determinan la ecuación básica de evolución del capital que maximiza el Valor Presente Neto:

$(10) \dot{I}=(r+\delta)(\phi / 2 \xi+I)-\phi / 2 \xi-\left[p(\alpha)-c_{q}^{\prime}\right](\gamma / 2 \xi)$

De modo que el stock de capital de estado estacionario viene dado por

( $C$ u a ndo $I=I=0)$

(1 1 ) $\left[r+\delta-\phi^{\prime} / \phi\right] \phi+\gamma c^{\prime} q=p \gamma$

En el gráfico A tenemos una representación del proceso de inversión y crecimiento de la empresa, y también podemos apreciar mejor cómo afectan a este proceso los parámetros propios del modelo. 
En el caso en que el mercado siempre es rentable y una vez elegido $\alpha^{*}$, la empresa desea llegar a $K^{*}$ y existe un único modo de hacerlo, sobre el sendero SS, dado el capital inicial $\boldsymbol{K} \boldsymbol{O}$ la firma define una inversión inicial en $\mathbf{I 0}$. El efecto de los costos de ajuste $\xi$ es cambiar la pendiente de SS y modificar la velocidad de convergencia a $K^{*}$ (pero no afectan a éste). El sendero AA sería el que recorrería una empresa con mayores costos de ajuste: la inversión inicial de $I$ sería menor.

En el caso colombiano, en el que el mercado no siempre es rentable una vez elegido $\alpha^{*}$, la empresa que desea llegar a $K^{*}$ tendrá un único modo de hacerlo, sobre el sendero SS, dado el capital inicial $\boldsymbol{K} \boldsymbol{0}$, pero si la firma define una inversión inicial en I0. El efecto de los costos de ajuste $\boldsymbol{\xi}$ nos determinan que debemos conservar la pendiente de SS y ajustar la velocidad de convergencia a $K^{*}$, de manera que no se presenten modificaciones al modelo. En este caso, una empresa con menores costos de ajuste recorrería el sendero (AA, KR); por lo tanto la inversión inicial de $I$ sería mayor, pero con mejores posibilidades de reintegro de la misma en un horizonte de tiempo menor.

\section{Gráfico A}

\section{Proceso de inversión y crecimiento esperado en una empresa regulada}

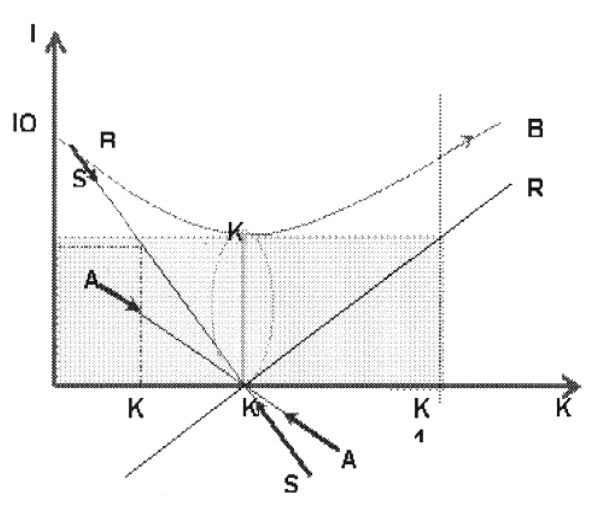

Como se puede observar en el grafico A, el valor presente neto de la empresa está siendo maximizado sobre los vértices SS y $\mathrm{AA}$; un vértice como $B B$ sólo sería elegido si la empresa tuviera que llegar a K1 en un periodo $\mathrm{Tb}$ exógenamente especificado, lo cual requiere, como se puede apreciar, aumentar necesariamente el nivel de inversión para lograr el propósito de manera más eficiente y eficaz dentro de un parámetro de equidad y justicia social.

Fuente: Elaborada por Abel Cano Morales 
Lógicas y dialécticas trascendentales de la inversión en sectores de infraestructura empresarial en Colombia

A continuación se podrá observar en el gráfico B que hay una representación del efecto de un cambio del costo de capital de la empresa, producto del aumento de $r$, el que disminuye el capital deseado de largo plazo (pero puede modificar además la velocidad de convergencia, aunque aquí se supone que no es así). En el caso de una empresa sujeta a regulación, el programa de maximización se realiza bajo algunas condiciones adicionales.

Téngase en cuenta que la privatización seguramente ha ayudado a aumentar el valor de $\gamma$ la productividad física media del capital) y a disminuir los costos operativos por incorporación de nuevas prácticas técnicas, operacionales y organizacionales (como nuevas estructuras internas de incentivos).

Asimismo, es preciso anotar que, tal y como se observa en el área bajo la curva, si las empresas reguladas recibieran incentivos tributarios, éstas efectuarían mayores inversiones en infraestructura y desarrollo tecnológico. Estas afirmaciones se pueden justificar en el desarrollo de las integrales propuestas, puesto que desde el punto de vista matemático existe una alta probabilidad que la inversión en términos financieros confluya de manera puntual hacia el desarrollo de empresas reguladas con mayores potencialidades de continuidad y crecimiento.

\section{Gráfico B \\ Representación del efecto de un cambio del costo de capital en una empresa regulada}

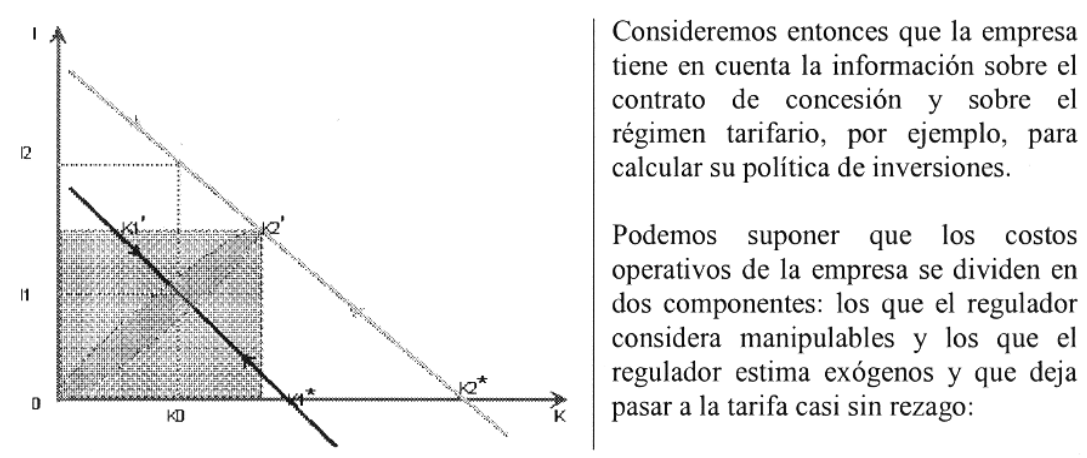

Fuente: Elaborada por Abel Cano Morales. 
Abel María Cano Morales

$$
C=p c(q, \alpha, E)+(1-p) c(q, \alpha)
$$

Donde $r$ es la proporción que se puede manipular y (1-r) la no que no es posible de manipulación. E representa un parámetro de esfuerzo de reducción de costos que la empresa puede manejar (como costos gerenciales, de consultoría). Para simplificar supondremos que la no manipulable puede escribirse como lineales en $\boldsymbol{q}$ (i.e. $(1-r) c q)$.

Además, el regulador podría reconocer una proporción $\mathrm{m}$ de los costos de inversión en la tarifa, en particular si se sobreimpone una condición de inversión mínima por periodo o de metas para alcanzar objetivos de cobertura. ${ }^{7}$

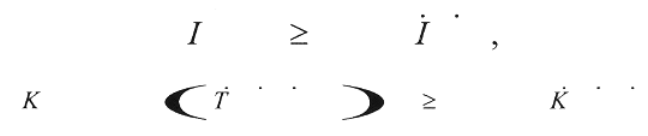

En este caso la tarifa esperada toma la forma:

$$
p=p_{0}+(1-p) c+\mu\left(\phi I+\xi I^{2}\right) / q
$$

Esto es, la empresa recupera una proporción (1-r) de sus costos operativos y un porcentaje $\mu$ de sus gastos de inversión. Como tiene un régimen de libre negociación o pass-through parcial, el costo de capital se reduce cuanto menor $r$ y cuanto mayor $\mu$, dado que cambia (disminuye) la $b$ de la inversión.

La extensión del programa $(T)$ también modifica el costo de capital porque cuanto más largo sea el contrato, tanto más incompleto.

En síntesis, parece que puede esperarse que el costo de capital se transforme en una función del coeficiente del poder, aquí r, de la proporción del costo de inversión compartida y del horizonte del contrato:

$$
r=r\left(r_{f}, E_{m}, p, \mu, T\right)
$$

\footnotetext{
7 Mecanismos de este tipo son los utilizados, por ejemplo, en agua donde el contrato de concesión original contemplaba un esquema donde los nuevos usuarios debían abonar un cargo de infraestructura que cubre los costos de capital de las inversiones asociadas a su suministro. Este sistema presentó graves problemas por cuanto el cargo de infraestructura mostró durante los primeros años una fuerte tasa de incobrabilidad, llevando finalmente a su sustitución por un cargo a todos los usuarios conectados.
} 
Lógicas y dialécticas trascendentales de la inversión en sectores de infraestructura empresarial en Colombia

Con la propiedad de que:

$$
r_{p}>0, r_{\mu}<0, r_{t}>0
$$

La consecuencia es que el costo de capital deviene una variable determinada en el contrato inicial. Así, por ejemplo, un régimen de precio supuesto o price-cap muy estricto que aumente $r$ determinará una $b$ más alta y afectará el factor de descuento del programa de optimización; la empresa requerirá una mayor compensación por riesgo más alto y reducirá el capital de largo plazo $K^{*}$ bajando la cobertura planeada por ella.

Por lo tanto el programa de optimización del valor presente esperado estará dado ahora por:

$$
\begin{aligned}
& V P N^{*}=\max \int_{0}^{T}\left[p_{0} q-p c(\alpha, q, E)-E-(1-\mu)\left(\phi I+\xi I^{2}\right)\right] e^{-r(p, \mu, T) t} d t+e^{-r(p, \mu, T) t} R \\
& R\left\{\left[K(T)-K_{0}\right](1-\mu)\right\}-K_{0} . \phi_{0}
\end{aligned}
$$

Sujeto a:

$\rightarrow \quad$ Condición de ritmo de inversión

$$
I \geq \bar{I}
$$

$\rightarrow \quad$ Condición de calidad

$$
\alpha \geq \bar{\alpha}
$$

$\rightarrow \quad$ Extensión del contrato por más tiempo

$$
T \geq \bar{T}
$$

$\rightarrow \quad$ Límites a la producción

$$
q \geq \bar{q} \text { o b iе } n K \quad(t) \geq \bar{K}(t) \forall t
$$

En la expresión del valor presente neto, el término

$$
+e^{-r T} R \quad\left\{[ \begin{array} { l l l } 
{ K } & { ( T ) - K _ { 0 } }
\end{array} ] \left(\begin{array}{lll}
1-\mu & -\mu
\end{array}\right.\right.
$$


Corresponde al valor residual de la firma que ésta recibe como compensación al final de la concesión. Notemos que depende del stock de capital final adelantado con fondos propios, y que se resta del $V P N$ el capital inicial adelantado (suponiendo que lo que se pagó por los activos estaba en relación con Ko). En el caso de una empresa privatizada es el valor de continuar el proceso de forma óptima. Para este caso particular supondremos que ese valor residual viene dado por:

$$
R \phi\left[K(T)-K_{0}\right](1-\mu) e^{-r t}
$$

Donde $R$ representa la proporción del Valor Residual que reconoce el mercado (en una serie infinita $R=R_{\delta}(r)$.

Igualmente observamos también que la condición de calidad mínima aquí es necesaria para que $\alpha>0$, y que se está suponiendo que la demanda no responde a los precios, sino que evoluciona de manera autónoma. Esa restricción no es necesaria para $e$, que será determinada por la empresa para minimizar sus costos

$$
\left(-p C_{e}^{\prime}=1\right)
$$

Está implícito, además, que la empresa no puede anticipar un valor presente máximo negativo si se intenta que participe en el negocio (condición de sostenibilidad). Veamos en el gráfico $\mathrm{C}$ el efecto de imponer algunas condiciones sobre el programa de inversiones y los dilemas que implica.

\section{Gráfico C \\ Representación del efecto de imponer algunas condiciones sobre el programa de inversiones en una empresa}

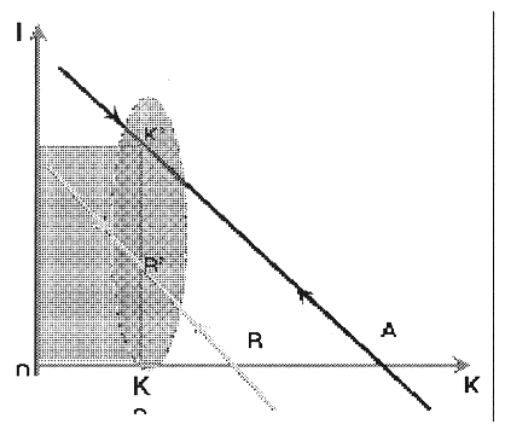

Un aumento de $r$, una menor cobertura de los costos operativos, hace que el stock de capital deseado de largo plazo se reduzca (de A a B) y que caiga la inversión (por aumento de costos operativos y de capital).

La forma de compensarlo es aumentando po, o eventualmente extender la concesión.

Fuente: Elaborada por Abel Cano Morales 
Lógicas y dialécticas trascendentales de la inversión en sectores de infraestructura empresarial en Colombia

En el gráfico D podemos observar lo que ocurre cuando el tiempo de concesión está dado en $T$ y la empresa no recupera el capital residual. Su política óptima será entonces no dejar instalado un stock de capital valioso. Recorrerá así el sendero $T T$, alcanzando un capital final nulo al final de la concesión.

Pero esta situación sólo será posible si el tiempo de concesión es por un periodo inferior a 5 años, pues el abanico de posibilidades de recuperar la inversión en una economía como la colombiana, donde el mercado de capitales es especulativo, sólo será posible recuperar la inversión en un tiempo no inferior a 20 años.

\section{Grafico D}

\section{Representación del efecto de imponer el tiempo de concesión a una empresa}

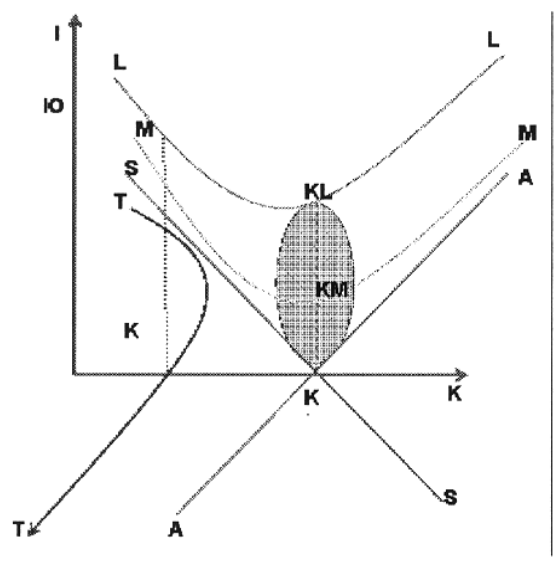

Para que el stock final fuera $\mathrm{K}^{*}$ en un tiempo de concesión finito, se requeriría imponer una restricción de capital final mínimo, de ritmo de inversión o bien definir un horizonte de concesión suficientemente largo como para que la empresa elija acercarse a $\mathrm{K}^{*}$ de propia voluntad (si una concesión es por 95 años, por ejemplo, nos pone sobre un sendero como $\mathrm{MM}$ ).

Fuente: Elaborada por Abel Cano Morales

El ritmo de inversión mínimo baja el valor presente de la empresa y le pone metas de avance que le incrementan sustancialmente los costos de ajuste de la inversión. El pass-through de costos de inversión, dado por $m$, compensa a la empresa al aumentar la pendiente del sendero SS; en principio no altera el capital deseado de largo plazo, excepto porque la firma no lo ve como capital residual valioso. 
Podemos simplificar un poco los cálculos para tener algunas comparaciones útiles. Partiremos del supuesto que cada uno de los operadores considera que el proceso de concesión se repite de manera indefinida, de modo que se puede quedar entonces con una proporción $R$ del valor residual del negocio.

Basándonos en la hipótesis de que la calidad y el esfuerzo $E$ han sido fijados exógenamente y no importan aquí, también admitamos que los bienes de capital no cambian de precio $(\phi=0)$, ya que las tarifas tampoco están sufriendo ningún tipo de indexación.

En ese caso especifico tendríamos que replantear la ecuación de inversión a la siguiente:

$$
(11) \dot{I}=[r(1-R)+\delta] \phi / 2 \xi+(r+d) I-\gamma\left[p_{0}-p C_{q}^{\prime}(\gamma K)\right] /(1-\mu) 2 \xi
$$

Como puede verse, (11) se obtiene tomando $R=\mu=0$ y $r=1$.

Por lo tanto, el estado estacionario estará entonces determinado por la siguiente ecuación:

$$
(12)[r(1-R)+\delta] \phi(1-\mu)+\gamma C^{\prime}{ }_{q} p=\gamma p_{0}
$$

Ecuación equivalente a (11) si se toman los valores de parámetros adecuados.

¿Qué diferencias se verían en el stock de capital de largo plazo de esta empresa según fueran $r, \mu$, y $R$ ?; es decir, si aumenta el coeficiente de poder de incentivos, la cobertura de largo plazo planeada por la firma baja. Esto se debe a que sube el costo marginal que la empresa debe enfrentar con sus propios recursos y a que el costo de capital aumenta (sube la $b$ del negocio). Si no queremos que $K^{*}$ caiga, el incremento de $r$ deberá ser acompañado por un aumento consistente de $p_{0}$. 
Lógicas y dialécticas trascendentales de la inversión en sectores de infraestructura empresarial en Colombia

\section{Mentefacto marco del modelo de inversión}

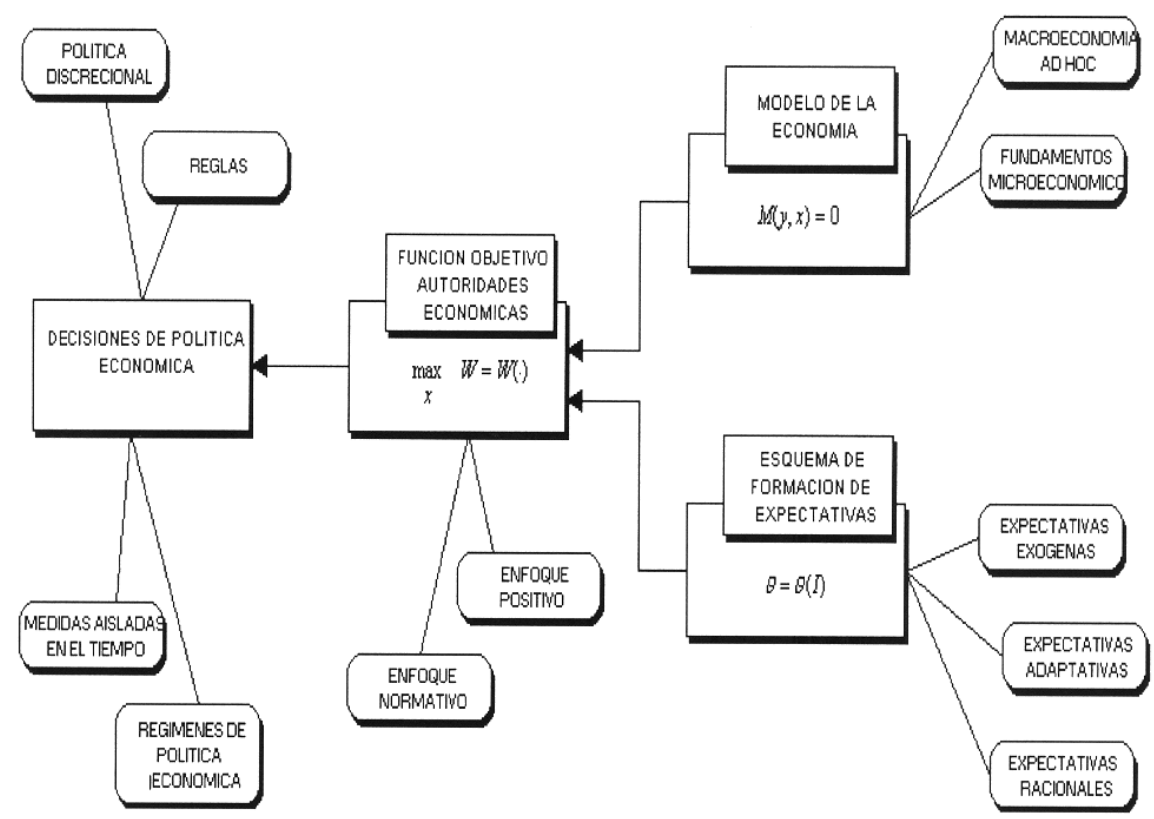

\section{Conclusiones}

La función objetivo de las autoridades económicas corresponde con el maximizado del modelo de política económica. Y tal y como su denominación indica, se trata de la función que representa las principales metas que las autoridades económicas intentan alcanzar. En la bibliografía de los textos especializados se supone implícitamente que las motivaciones de la pluralidad de entes decisionales que intervienen en la esfera de la política económica son representables a través de una única función de utilidad que se asocia a un agente ficticio llamado gobierno, policy maker o, simplemente, autoridades económicas. En el ámbito concreto de la literatura normativa (esto es, el que ampliamente ha dominado en la literatura) 
se supone, además, que la función objetivo del modelo de política económica es el resultado de agregar también las preferencias del conjunto de los agentes privados de la economía. No obstante, salvo en los modelos de agente (privado) representativo (entendidos éstos como sinónimo de modelos de agentes idénticos), donde el proceso de agregación es trivial bajo el usual supuesto de homogeneidad de las funciones de utilidad de los individuos, no suele explicitarse en la literatura las condiciones que garantizan la factibilidad de estos procesos de agregación de preferencias, tanto en el ámbito del sector privado como del sector público.

En la teoría de la política económica se distingue entre variables objetivo (y) y variables instrumento, $x$. Las primeras representan aquellas metas que el policy maker interpreta como "objetivos intermedios" al servicio del bienestar de los agentes, mientras que las segundas son las variables que están bajo el control de las autoridades y que se emplean para maximizar la función objetivo. Son múltiples las clasificaciones disponibles de los objetivos e instrumentos de política económica (véase Kirschen et alia, 1964, para un tratamiento taxonómico de los objetivos e instrumentos de política). En la literatura tradicional las variables objetivo se definen frecuentemente en términos de desviaciones (normalmente cuadráticas) con respecto a los valores óptimos de ciertas variables representativas de males macroeconómicos (de modo preferente, la inflación y el desempleo), lo que convierte al problema de optimización de las autoridades en un problema de minimización y a la función objetivo en una función de pérdidas. Es también práctica común en esta rama de la literatura la consideración de las variables objetivo como argumentos de la función objetivo de las autoridades, a diferencia de la moderna literatura en la que predomina la consideración de las funciones de utilidad de los agentes como tales argumentos.

El modelo $M$ es la primera de las dos restricciones que cabe incluir en un modelo de política económica. Se trata del considerado por las autoridades económicas como el verdadero modelo de la economía. Recoge el conjunto de ecuaciones que representa el funcionamiento global de la economía (resultante de la interacción de múltiples agentes privados) sobre la que se aplica la política económica. Su específico formato es consecuencia de la estrategia modelizadora elegida por el economista. (Es bien sabido, y debe recordarse por la influencia que la estrategia modelizadora tiene en la concepción de la política económica, que la macroeconomía nos ha deparado a lo largo de su historia un amplio conjunto de modelos macroeconómicos (véase Bitrán Muñoz, 1997). 
Lógicas y dialécticas trascendentales de la inversión en sectores de infraestructura empresarial en Colombia

El modelo de la economía puede consistir en un conjunto de ecuaciones descriptivas de las reglas de decisión de los agentes privados (enfoque tradicional) o un sistema de ecuaciones que recogen las condiciones de equilibrio general de una economía; es decir, las condiciones de optimalidad individual y de vaciado de mercado (enfoque de la moderna teoría de la política económica). Adecuadamente reformulado, el modelo expresa la relación existente entre el subconjunto de variables endógenas que representan los objetivos de las autoridades y aquellas variables exógenas que las autoridades utilizan como instrumentos de política económica. El modelo permite, por tanto, estudiar la coherencia entre objetivos e instrumentos de una determinada política económica — es decir, de una determinada especificación del vector de instrumentos - y cuantificar los efectos desplegados por las variaciones del vector de instrumentos sobre el vector de objetivos.

El esquema de formación de expectativas de los agentes constituye la otra restricción fundamental por considerar en un modelo de política económica. Este componente del modelo de política económica sirve de expresión del volumen de información que condiciona los procesos de decisión de los agentes y del grado de eficiencia con que dicha información es empleada por los agentes. El esquema de formación de expectativas es, por tanto, una función — que admite distintas especificaciones, según la hipótesis de formación de expectativas - del conjunto de información de los agentes, $I$. Aunque esta función forma parte del problema de optimización del que se derivan las reglas de decisión de los agentes, podría argumentarse que se trata de un elemento redundante del modelo de política económica, optaremos por su consideración explícita y diferenciada del modelo de la economía debido a la importancia que en el estudio de las posibilidades y limitaciones de la política económica han tenido per se los distintos esquemas de formación de expectativas por parte del público.

El análisis de determinantes de inversión resulta, a la vez de interesante, complicado por dos razones: la primera es por el deterioro de las condiciones (o determinantes estructurales) de demanda y rentabilidad; segunda, el análisis de determinantes se dificulta debido a la convergencia de varios shocks y la combinación de la operación de diversos tipos de rezagos con la escasez de observaciones posteriores a esos shocks, es decir, para el periodo de apertura). Situación que se complica un poco más cuando se trata de empresas de servicios regulados. 
Abel María Cano Morales

\section{Bibliografía}

ABETTI, PIER A., Linking Technology and Bussiness Strategy, American Management Association, New York, 1999.

ALEXANDER Ian, Colin Mayer y Helen Weeds: Regulatory Structure Risk and Infraestructure Firms, Policy Research Working Paper 1998-The World Bank - Private Sector Development Department December 1996. Banco Mundial: Las reformas estructurales y la inversión privada en áreas de infraestructura (LC/L.1083), Chile, Imprenta nacional, Santiago, noviembre de 1997.

ACOSTA MEDINA, Amilkar David, Ajuste fiscal o desajuste estructural. De la luna de miel a la luna de hiel, Imprenta Nacional de Colombia, Santa Fe de Bogotá, 1999, 346 pp.

AMAT, Oriol, Un Nuevo enfoque para optimizar la gestión empresarial, motivar a los empleados y crear valor, Grupo Editorial Norma, Cali Valle, Colombia, 1999, $223 \mathrm{pp}$.

ARGOS, Manuel R, Inversión extranjera directa en América Latina: su contribución al desarrollo, Fondo de Cultura Económica, México, 1996, 209 pp.

ARMSTRONG, M., "Network Interconnection", Department of Economics, University of Southamptom, Mc Graw Hill Interamericana, Madrid, 1996, 275 pp.

AUER BACK, Alan y Laurence Kotlikoff, "Fiscal policy, saving, and Growth" Macroeconomics an integrated Approach, Institute of Technology, Massachusetts, 1978.

BACIDORE, J.M. et al., "The Search for the Best Financial Performance Measure", Financial Analysts Journal. May/June, Massachusetts, 1997, pp. 11-20.

BARRO, R., Macroeconomía: teoría y política, Mc Graw Hill Interamericana, Santa Fe de Bogotá, 1997. 
Lógicas y dialécticas trascendentales de la inversión en sectores de infraestructura empresarial en Colombia

BELL, A. "Recent Development in Interconnection arrangements in UK telecommunications", Revista Internazionale di Scienze Sociali, Anno CIII No.1 gennaio-marzo, Palermo, Italia, 1995.

BITRÁN, MUÑOZ, Eduardo, Hacia el estado regulador. CIEPLAN. La gestión privada y la inversión en el sector eléctrico chileno (LC/L.1070), Documento de trabajo, Imprenta Nacional de Chile, Santiago, septiembre, 1997, $246 \mathrm{pp}$.

BLUMENFELD, A. y J. COHEN, "Overview of the Telecommunication Act of 1996", International Training Program on Utility Regulation and Strategy, University of Florida, 13 al 24 de enero, Marylan, USA, 1997.

CANO MORALES, Abel María, "Ponencia sobre el EVA (Valor Económico Agregado) vs. TOC (La Teoría de Restricciones), en Congreso de Economistas Empresariales. Facultad de Economía Empresarial, Universidad Autónoma de Manizales, Manizales, 2 mayo, 2000.

CANO MORALES, Abel María y Jahir Alexander Gutiérrez Ossa, "Análisis del crecimiento económico desde las perspectivas determinantes del ahorro y la inversión en Colombia", en Avances en investigación económica, administrativa y contable, sello editorial Universidad de Medellín, pp. 311-332, 2005.

CÁRDENAS, Santa María, "Flujos de capital e inversión en los modelos de crecimiento endógeno. Un análisis empírico para 81 países desarrollados", en revista Coyuntura Económica 1, pp. 113-137. Santa fe de Bogotá, abril, 1992.

CEPAL (Comisión Económica para América Latina y el Caribe), Anexo estadístico del balance preliminar de las economías de América Latina y el Caribe, varios números, Banco de la República. Santa fe de Bogotá, 2002.

CLAVIJO, Sergio, Política fiscal y Estado en Colombia, Ediciones Universidad de los Andes, Bogotá, 1998. 
CORTS, K. (1995), "Regulation of a multiproduct monopolist: effects on pricing and bundling", Journal of Industrial Economics, XLIII No. 4, pp. 377-397.

CHÁVEZ J.A. y M.G. Budedo: "Logros y retos de las finanzas públicas en México", Revista de la CEPAL, No.112, Santiago de Chile, junio 2000.

DANE (Departamento Administrativo Nacional de Estadísticas), Estadísticas fiscales, de los años 1975 al 2002, Santa fe de Bogotá.

DIAN (Dirección de Impuestos y Aduanas Nacionales), Información estadística, de los años 1975 al 2002, Santa fe de Bogotá.

DÍEZ DE CASTRO, L., y J. López Pascual, Dirección financiera, Prentice Hall, Madrid, 2001.

DNP (Departamento Nacional de Planeación), "Indicadores de coyuntura", en Finanzas Públicas, Bogotá, varios números, desde los años 1986 al 2002.

DOYLE, C., "A case against access charge", Rivista Internazionale di Scienze Sociali, Anno CIII No.1 gennaio-marzo, 1995.

EATON, B. y R. Lipsey (1989), "Product Differentation", Schmalensee, R y Willig, R. eds., Handbook of Industrial Organization, Volume I, Cap. 12 North Holland, 1989, pp. 723-768.

ESTABILIZACIÓN Y CRECIMIENTO. Nuevas lecturas de macroeconomía colombiana, Compilador Roberto Steiner, Tercer Mundo Editores. Fedesarrollo, Santa Fe de Bogotá, 1994, pp. 249.

ENDERS, W., Applied Econometric Time Series, John Wiley \& Sons, London, 1995.

ESLAVA MEJÍA, Marcela, "Crisis y recuperación de las finanzas públicas: lecciones de América Latina para el caso colombiano", revista Archivos de Macroeconomía, DNP, Bogotá, julio, 1999. 
Lógicas y dialécticas trascendentales de la inversión en

sectores de infraestructura empresarial en Colombia

GRAHAM, C., "Universal Service: a comparative perspective", Access rights and affordability: the social agenda for regulation of utilities charging policy, Proceedings CIPFA-CRI, Massachusetts, 1995.

GARCIA-GUTIÉRREZ, Carlos, Juan Mascareñas y Eduardo Pérez, Casos prácticos de inversión y financiación en la empresa, Pirámide, Madrid, 1998, $350 \mathrm{pp}$.

HENDERSON, J. M. y R. E. Quandt, Teoría microeconómica, tercera edición, Editorial Ariel, Barcelona, 1985.

HARBERGER A.C., "On the Use of Distributional Weights in Social Cost-Benefit Analysis", Journal of Political Economy, vol.86, No. 2, 1978, pp. 87-120.

HAY, D. y D. MORRIS, Industrial Economics and Organization: Theory and Evidence, segunda edición, Oxford University Press, 1991.

HUNGARIAN COMM, Auth, "Hungarian telecommunications: Regulatory environment and authority", International Training Program on Utility Regulation and Strategy, University of Florida, 13-enero, 1997.

HUTCHINSON, Michael, "Telecommunications Reform in Australia", Implementing Reforms in the Telecommunications Sector: Lessons from experience, The World Bank, 1994.

KALMANOVITZ, Salomón, Economía y nación: Una breve historia de Colombia, cuarta edición, TM editores, Santa Fe de Bogotá,1997, pp. 457.

LAFFONT, J, y J. TIROLE (1993), A Theory of Incentives in Procurement and Regulation, MIT Press, 1993.

MASCAREÑAS, Juan y Gustavo Lejarriaga, Análisis de proyectos de inversión, Eudema, Madrid, 1992, 120 pp.

STEWART, G. Bennett, The Quest for Value: the EVA management guide for Senior Managers, HarperCollins, University of Florida, Marylan USA, 15 al 31 agosto, 1999. (A) 